ESAIM: Proceedings, Vol. 4, 1998, 137-152

Contrôle et Équations Aux DÉRivées Partielles

http://www.emath.fr/proc/Vol.4/

\title{
REMARKS ON ROBUSTNESS OF STABILITY WITH RESPECT TO THICKNESS FOR ELASTIC STRUCTURES
}

\author{
MARY ANN HORN ${ }^{1}$ \\ Department of Mathematics \\ 1326 Stevenson Center \\ Vanderbilt University \\ Nashville, TN 37240
}

Key Words : Uniform stabilization, robustness, elastic systems, boundary controllability, feedback control.

AMS Subject Qualification : 35L55, 35Q72, 73C02, 93B52, 93D15.

\footnotetext{
${ }^{1}$ Partially supported by National Science Foundation Grant NSF DMS-9501051.

Article published by EDP Sciences and available at http://www.edpsciences.org/proc or http://dx.doi.org/10.1051/proc:1998025
} 


\begin{abstract}
Models of elastic systems are often inherently dependent upon small parameters such as thickness. Using the results on the original model to obtain information on the system as the thickness tends to zero can be far from straightforward. An illustration of stability results and discussion of the difficulties that arise in deriving stability for the limiting system are presented.
\end{abstract}




\section{Introduction}

Modelling of elastic structures often inherently depends on small parameters. For example, the term corresponding to rotational inertia in the Kirchhoff plate model is directly proportional to the square of the thickness of the plate. While the thickness is fixed, it is assumed to be small, even negligible, in comparison to the width and breadth of the plate. Thus, a number of questions naturally arise. If the terms which are proportional to thickness are neglected, is the resulting model well-posed? If the original model can be stabilized via boundary feedback, will the same feedback stabilize the limiting system when the thickness tends to zero?

Neither of these two questions has an obvious answer. To illustrate, consider the following model of a Kirchhoff plate with homogeneous Dirichlet boundary conditions and a control acting as a moment through a second order boundary condition. Let $\Omega$ be an open bounded domain in $\mathbb{R}^{2}$ with sufficiently smooth boundary, $\partial \Omega$. In $\Omega$, consider the Kirchhoff plate model:

$$
\begin{array}{rcc}
w_{t t}-\gamma \Delta w_{t t}+\Delta^{2} w=0 & \text { in } & Q_{T} \equiv \Omega \times(0, T) \\
w(0, \cdot)=w_{0}, & w_{t}(0, \cdot)=w_{1} & \text { in } \Omega \\
w=0 & \text { on } & \Sigma_{T} \equiv \partial \Omega \times(0, T) \\
\Delta w=u & \text { on } \Sigma_{T}
\end{array}
$$

where the parameter $0<\gamma<<1$ is proportional to the square of the thickness and is therefore assumed to be small. It is clear that the corresponding limit problem is described by the following model, sometimes referred to as the EulerBernoulli plate (see [10]):

$$
\begin{array}{rc}
w_{t t}+\Delta^{2} w=0 & \text { in } Q_{T} \\
w(0, \cdot)=w_{0}, \quad w_{t}(0, \cdot)=w_{1} & \text { in } \Omega \\
w=0 & \text { on } \Sigma_{T} \\
\Delta w=u & \text { on } \Sigma_{T}
\end{array}
$$

Already, critical differences appear between the models. The first is hyperbolic with finite speed of propagation. The second is Petrovsky type with infinite speed of propagation. Additionally, as one would expect, optimal regularity results for the two systems give solutions which belong to very different spaces: For the first system,

$$
u \in L^{2}\left(\Sigma_{T}\right) \Longrightarrow\left(w, w_{t}\right) \in C\left([0, T] ; H^{2}(\Omega) \cap H_{0}^{1}(\Omega) \times H_{0}^{1}(\Omega)\right),
$$

(see [7]), while for the corresponding limit system,

$$
u \in L^{2}\left(\Sigma_{T}\right) \Longrightarrow\left(w, w_{t}\right) \in C\left([0, T] ; H_{0}^{1}(\Omega) \times H^{-1}(\Omega)\right),
$$

(see $[8])$. Thus, the above topologies are a natural choice in which to address the questions of controllability and stabilization. However, it is apparent that 
if the second system is to be thought of as the limit of the first, the space to consider is not the one given by optimal regularity results, but rather

$$
\left(w, w_{t}\right) \in C\left([0, T] ; H^{2}(\Omega) \cap H_{0}^{1}(\Omega) \times L^{2}(\Omega)\right) .
$$

\subsection{Literature for the Kirchhoff plate}

In both of the above models, stabilization has been addressed independently. A number of results directly related to these models include:

i.) Uniform stabilization of model (1.1) with $u=-\frac{\partial}{\partial \nu} w_{t}$ in the space $H^{2}(\Omega) \cap$ $H_{0}^{1}(\Omega) \times H_{0}^{1}(\Omega)$.

Critical assumption: The domain $\Omega$ must be convex (see [7]).

ii.) Uniform stabilization of model (1.1) with $u=-\frac{\partial}{\partial \nu} w_{t}$ in the space $H^{2}(\Omega) \cap$ $H_{0}^{1}(\Omega) \times H_{0}^{1}(\Omega)$.

No strong geometric assumptions on the domain (see [3]).

iii.) Uniform stabilization of model (1.2) with $u=-\frac{\partial}{\partial \nu} w_{t}$ in the space $H^{2}(\Omega) \cap$ $H_{0}^{1}(\Omega) \times L^{2}(\Omega)$.

Critical assumption: The domain $\Omega$ must be a sphere or a small deformation of a sphere (see $[6])$.

$i v$.) Uniform stabilization of model (1.2) with $u=-\frac{\partial}{\partial \nu} w_{t}$ in the space of optimal regularity $H_{0}^{1}(\Omega) \times H^{-1}(\Omega)$.

No strong geometric assumptions on the domain (see [2] and [5]).

In attempting to address the question of stabilization simultaneously for both systems, the feedback control must satisfy two goals:

i.) Provide exponential decay rates for the first Kirchhoff plate model (1.1) which are independent of the parameter $\gamma>0$;

ii.) Not require any geometric restrictions on the domain $\Omega$ except for the usual regularity hypothesis.

Thus, the least restrictive of the geometric assumptions required in the previous literature is assumed, yet by achieving the first criteria, stability results for both systems (1.1) and (1.2) can be deduced simultaneously.

\section{The Feedback System and Uniform Stabiliza- tion}

In [4], it is precisely the two above goals which are attained. To facilitate discussion of these results, it is necessary to define the appropriate energy for each of the two systems. As stated earlier, the regularity results in [7] indicate that the appropriate space for stabilization of system $(1.1)$ is $H^{2}(\Omega) \cap H_{0}^{1}(\Omega) \times$ 
$H_{0}^{1}(\Omega)$ with a suitable topology. For the model, the energy is defined to be

$$
\begin{aligned}
E_{\gamma}(t) & \equiv\left\|w_{t}(t)\right\|_{L^{2}(\Omega)}^{2}+\gamma\left\|\nabla w_{t}(t)\right\|_{L^{2}(\Omega)}^{2}+\|\Delta w(t)\|_{L^{2}(\Omega)}^{2} \\
& \equiv\left\|w_{t}(t)\right\|_{H_{0, \gamma}^{1}(\Omega)}^{2}+\|\Delta w(t)\|_{L^{2}(\Omega)}^{2}
\end{aligned}
$$

where $H_{0, \gamma}^{1}(\Omega)$ will henceforth indicate the Hilbert space $H_{0}^{1}(\Omega)$ with norm

$$
\|g\|_{H_{0, \gamma}^{1}(\Omega)}^{2} \equiv\|g\|_{L^{2}(\Omega)}^{2}+\gamma\|\nabla g\|_{L^{2}(\Omega)}^{2}
$$

which is equivalent to the usual norm. Notice that with this definition of energy, if no control is implemented, i.e., if $u \equiv 0$, the resulting system is conservative.

Similarly, the energy for system (1.2) is given by

$$
E_{0}(t) \equiv\left\|w_{t}(t)\right\|_{L^{2}(\Omega)}^{2}+\|\Delta w(t)\|_{L^{2}(\Omega)}^{2},
$$

which corresponds directly to the energy of system (1.1) when $\gamma=0$. Notice that the inclusion of rotational inertia results in an increase in regularity for the velocity. This boost in regularity is an advantage in obtaining stability estimates for system (1.1) (see [3]). The fact that $w_{t} \in L^{2}(\Omega)$ for system (1.2) adds technical difficulties which must be overcome. Thus, by considering system (1.2) to be the limit of system (1.1), the proof of stabilization for both systems may take advantage of the increased regularity of system (1.1), at least up to a point.

To uniformly stabilize the two systems, the control is defined by $u \equiv-\frac{\partial}{\partial \nu} w_{t}$. This choice of feedback has been well established as a reasonable candidate for stabilization of the first system (see $[4,7]$ ). With this definition of $u$, the following wellposedness and regularity results are valid.

Theorem $2.1[7]$

i.) Wellposedness of system (1.1) on $H^{2}(\Omega) \cap H_{0}^{1}(\Omega) \times H_{0}^{1}(\Omega)$.

Let $\left(w_{0}, w_{1}\right) \in H^{2}(\Omega) \cap H_{0}^{1}(\Omega) \times H_{0}^{1}(\Omega)$. Then there exists an unique solution (in the sense of distributions), $\left(w(t), w_{t}(t)\right) \in C\left([0, T] ; H^{2}(\Omega) \cap\right.$ $\left.H_{0}^{1}(\Omega) \times H_{0}^{1}(\Omega)\right)$, satisfying system (1.1) with $u=-\frac{\partial}{\partial \nu} w_{t}$.

ii.) $L^{2}$-boundedness of the feedback operator.

For $\left(w_{0}, w_{1}\right) \in H^{2}(\Omega) \cap H_{0}^{1}(\Omega) \times H_{0}^{1}(\Omega)$, the solution, $w(t)$, of system (1.1) with $u=-\frac{\partial}{\partial \nu} w_{t}$ satisfies the inequality:

$$
\int_{0}^{T}\left\|\frac{\partial}{\partial \nu} w_{t}\right\|_{L^{2}(\partial \Omega)}^{2} d t \leq E_{\gamma}(0)
$$

Corresponding wellposedness results for the limiting system have been proven in [8] and are given in the following theorem.

ESAIM: Proc., Vol. 4, 1998, 137-152 
Theorem 2.2 [8]

i.) Wellposedness of system (1.2) on $H^{2}(\Omega) \times L^{2}(\Omega)$.

Let $\left(w_{0}, w_{1}\right) \in H^{2}(\Omega) \times L^{2}(\Omega)$. Then there exists an unique solution (in the sense of distributions), $\left.\left(w(t), w_{t}(t)\right) \in C\left([0, T] ; H^{2}(\Omega) \times L^{(} \Omega\right)\right)$, satisfying system (1.2) with $u=-\frac{\partial}{\partial \nu} w_{t}$.

ii.) $L^{2}$-boundedness of the feedback operator.

For $\left(w_{0}, w_{1}\right) \in H^{2}(\Omega) \times L^{2}(\Omega)$, the solution, $w(t)$, of system (1.2) with $u=-\frac{\partial}{\partial \nu} w_{t}$ satisfies the inequality:

$$
\int_{0}^{T}\left\|\frac{\partial}{\partial \nu} w_{t}\right\|_{L^{2}(\partial \Omega)}^{2} d t \leq E_{0}(0)
$$

With the above wellposedness and regularity results, it is appropriate to turn to the question of stabilization. Uniform stabilization results for the two systems can be stated as follows.

Theorem 2.3 [4] Uniform stabilization of system (1.1).

The feedback system (1.1) with $u=-\frac{\partial}{\partial \nu} w_{t}$ is uniformly (exponentially) stable with respect to the initial energy on the space $H^{2}(\Omega) \cap H_{0}^{1}(\Omega) \times$ $H_{0}^{1}(\Omega)$. I.e., there exist constants, $C>0$ and $\omega>0$, such that

$$
E_{\gamma}(t) \leq C e^{-\omega t} E_{\gamma}(0)
$$

Moreover, the constants $C$ and $\omega$ are independent of $\gamma$.

Corollary 2.1 [4] Uniform stabilization of system (1.2).

The feedback system (1.1) with $u=-\frac{\partial}{\partial \nu} w_{t}$ is uniformly (exponentially) stable with respect to the initial energy on the space $H^{2}(\Omega) \times L^{2}(\Omega)$. I.e., there exist constants, $C>0$ and $\omega>0$, such that

$$
E_{0}(t) \leq C e^{-\omega t} E_{0}(0)
$$

To prove Theorem 2.3, the challenges are numerous. Through the use of semigroup theory, it is straightforward to show that the proof of Theorem 2.3 follows directly after establishing the following stability estimate.

Lemma 2.1 (Stability Estimate.) Let $T$ be sufficiently large. If $w(t)$ is the solution of system (1.1) with $u=-\frac{\partial}{\partial \nu} w_{t}$, then there exists a constant $C(T)$ which is independent of $\gamma$, such that $w(t)$ satisfies the inequality,

$$
E_{\gamma}(T) \leq C(T)(1+\gamma)\left\|\frac{\partial}{\partial \nu} w_{t}\right\|_{L^{2}\left(\Sigma_{T}\right)}^{2} .
$$

Tracking the dependence of $\gamma$ in the proof of this lemma creates many difficulties. For this discussion, the focus will be on two particular issues: Trace regularity results and the removal of lower-order terms through a compactness/uniqueness argument. 


\section{$2.1 \quad$ Trace Regularity}

Sharp trace regularity results have been frequently used to obtain stability estimates which are free of the strong geometric constraints commonly associated with uniform stability. Derivation of these regularity results is based on microlocal analysis which translates information for the symbol of the differential operator into estimates for the solution. While a complete discussion may be found in [4], a few important facts should be noted here. One estimate needed in the proof of the stability estimate (2.7) is the following.

Lemma 2.2 [4] (Trace Regularity.) Let $w(t)$ be the solution to system (1.1) with $u=-\frac{\partial}{\partial \nu} w_{t}$ and let $0<\alpha<T / 2$. Then $w(t)$ satisfies the inequality:

$\left\|\frac{\partial}{\partial \tau} \frac{\partial}{\partial \nu} w\right\|_{L^{2}(\alpha, T-\alpha ; \partial \Omega)}^{2} \leq C\left\{\left\|\frac{\partial}{\partial \nu} w_{t}\right\|_{L^{2}\left(\Sigma_{T}\right)}^{2}+\|w\|_{L^{2}\left(0, T ; H^{2-\epsilon}(\Omega)\right.}^{2}\right\}$,

where $0<\epsilon<\frac{1}{2}$ and $C$ is independent of $\gamma$.

Such trace estimates are highly dependent on the boundary conditions considered in systems (1.1) and (1.2). In fact, a related result is obtained in [9]. However, a different set of higher order boundary conditions is considered.

Sketch of the Proof of Lemma 2.2: Via a partition of unity and a flattening of the boundary procedure, it can be established that the proof of Lemma 2.2 is equivalent to proving the same lemma for the following problem.

Let $\Omega=\{(x, y) ; x \geq 0\}, \partial \Omega=\{(x, y) ; x=0\}$. Let $w(t)$ be the solution to the system:

$$
\begin{array}{rll}
\mathcal{P} w=f & \text { in } & Q_{T} \\
w=g_{1} & \text { on } & \Sigma_{T} \\
\mathcal{B} w=g_{2} & \text { on } & \Sigma_{T}
\end{array}
$$

where the operator $\mathcal{P}$ (modulo lower order terms) and the boundary operator $\mathcal{B}$ (modulo lower order terms) are defined by:

$$
\begin{gathered}
\mathcal{P}\left(x, y ; D_{t}, D_{x}, D_{y}\right) \\
\equiv-a(x, y) D_{t}^{2}+\gamma a(x, y) D_{t}^{2}\left(a_{1}(x, y) D_{y}^{2}+2 a_{2}(x, y) D_{x} D_{y}+D_{x}^{2}\right) \\
\quad+\left(a_{1}(x, y) D_{y}^{2}+2 a_{2}(x, y) D_{x} D_{y}+D_{x}^{2}\right)^{2}
\end{gathered}
$$$$
\text { and } \mathcal{B}\left(x, y ; D_{t}, D_{x}, D_{y}\right) \equiv a_{1}(x, y) D_{y}^{2}+2 a_{2}(x, y) D_{x} D_{y}+D_{x}^{2} \text {. }
$$

In the above definitions, $a(x, y)>0, a_{j}(x, y)$ depend smoothly on $(x, y)$ for $j=$ 1,2 , are constant outside a compact set, and satisfy the appropriate ellipticity conditions:

$$
\begin{aligned}
& a_{1}(x, y) \geq \rho>0 \quad \forall(x, y) \in \Omega \\
& a_{1}(x, y)-a_{2}^{2}(x, y) \geq \rho>0
\end{aligned}
$$

Additionally, the notation, $D_{z} \equiv \frac{1}{i} \frac{\partial}{\partial z}$ has been adopted.

For this more general problem, the following proposition holds:

ESAIM: Proc., Vol. 4, 1998, 137-152 
Proposition 2.1 Let $w(t) \in H^{2}\left(Q_{T}\right)$ be the solution to (2.9). Then $w(t)$ satisfies the estimate:

$$
\begin{aligned}
\left\|\frac{\partial}{\partial \tau} \frac{\partial}{\partial \nu} w\right\|_{L^{2}(\alpha, T-\alpha ; \partial \Omega)}^{2} \leq\left\{\| \frac{\partial}{\partial \nu} w_{t}\right. & \left\|_{L^{2}\left(\Sigma_{T}\right)}^{2}+\right\| g_{1} \|_{H^{1}\left(0, T ; L^{2}(\partial \Omega)\right)}^{2} \\
+ & \left\|g_{1}\right\|_{L^{2}\left(0, T ; H^{2}(\partial \Omega)\right)}^{2}+\left\|g_{2}\right\|_{L^{2}\left(\Sigma_{T}\right)}^{2} \\
& \left.+\|f\|_{H^{-3 / 2+\epsilon}(\Omega)}^{2}+(1+\gamma)\|w\|_{L^{2}\left(0, T ; H^{2-\epsilon}(\Omega)\right.}^{2}\right\}
\end{aligned}
$$

where $0<\alpha<T / 2,0<\epsilon<\frac{1}{2}$ and the constant $C$ is independent of $\gamma$.

A crucial factor in the ability to track $\gamma$ is that the parameter only multiplies the mixed derivative, both in the original problem and after the transformation to the half-space. This can be seen as well in the symbol of the operator $\mathcal{P}$ which, with the above definition in (2.10), is given by

$$
\wp(x, y ; s, \xi, \eta)=-a s^{2}+\gamma a s^{2}\left(a_{1} \eta^{2}+a_{2} \xi \eta+\xi^{2}\right)+\left(a_{1} \eta^{2}+a_{2} \xi \eta+\xi^{2}\right)^{2} .
$$

In the proposition, the tangential derivative must be eliminated. If it could simply be interchanged with the time derivative, the result would be a bound in terms of the norm of the control. Clearly, this is too much to hope. However, this is motivation to focus on the behavior of the symbol $\wp$ as $s$ and $\eta$, respectively corresponding to the time and tangential derivatives, change in value.

Basically, the idea is to "microlocalize" the problem. Smooth cutoff functions must be used to eliminate possible problems near the origin, i.e., near $s=\eta=0$, and to decompose the $s \eta$-plane into "elliptic" and "nonelliptic" regions. The cutoff near the origin results in the shortening of the time interval from $(0, T)$ to $(\alpha, T-\alpha)$. Two cases arise from the decomposition of the $s \eta$-plane. If $|\eta| \geq 2 k_{e}|s|$, where $k_{e}$ is sufficiently small, the symbol can be shown to be elliptic of order four. Thus, standard elliptic estimates apply in this region and the dependence on $\gamma$ can be easily seen by studying the symbols of resulting commutators of $\mathcal{P}$ and $\mathcal{B}$ with the cutoff functions. If $|\eta| \leq k_{e}|s|$, then the definition of the symbol as the transform of the derivative can be used to directly bound the norm of the tangential derivative by the norm of the time derivative with no dependence on $\gamma$. This results in a bound of the norm of the second order trace in Proposition 2.1 in terms of the norm of the control. Combining the results in the two regions gives the desired estimate, (2.12).

\subsection{Elimination of Lower Order Terms}

Compactness/uniqueness arguments are often used during the final stages of the proof of the stability estimate (2.7). Such arguments show that the terms which are of lower order in comparison to the energy norm may be bounded by the norm of the control. Multiplier methods together with sharp trace regularity estimates combine to prove: 
Lemma 2.3 Let $T$ be sufficiently large and $w(t)$ be the solution to system (1.1) with $u=-\frac{\partial}{\partial \nu} w_{t}$. Then $w(t)$ satisfies the estimate:

$E_{\gamma}(T) \leq C(T)(1+\gamma)\left\{\left\|\frac{\partial}{\partial \nu} w_{t}\right\|_{L^{2}\left(\Sigma_{T}\right)}^{2}+\|w\|_{C\left([0, T] ; H^{2-\epsilon}(\Omega)\right)}^{2}\right\}$,

where $0<\epsilon<\frac{1}{2}$ and $C(T)$ is independent of $\gamma$.

Thus, all that remains is to eliminate the second term on the right hand side of this inequality. At issue is the fact that this is done using a proof by contradiction.

The following proposition can be proven by following arguments that have become well known in establishing stability, taking advantage of the regularity of the solutions and of unique continuation results for system (1.1) with overdetermined homogeneous boundary conditions.

Proposition 2.2 [4] Assume $w(t)$ is the solution to system (1.1) with $u=$ $-\frac{\partial}{\partial \nu} w_{t}$. Then $w(t)$ satisfies the inequality:

$$
\|w\|_{C\left([0, T] ; H^{2-\epsilon}(\Omega)\right)}^{2} \leq C(T, \gamma)\left\|\frac{\partial}{\partial \nu} w_{t}\right\|_{L^{2}\left(\Sigma_{T}\right)}^{2} \quad \text { for } 0<\epsilon<\frac{1}{2} .
$$

Unfortunately, there is no information on the behavior of $C(T, \gamma)$ beyond the fact that it is bounded for fixed $T$ and $\gamma$. Thus, further work must be done to insure that $C(T, \gamma)$ is actually independent of $\gamma$ which implies, in particular, that the above estimate is uniform for all $\gamma>0$. Therefore, the following lemma must be proven.

Lemma 2.4 Let $w(t)$ be the solution to system (1.1) with $u=-\frac{\partial}{\partial \nu} w_{t}$. Then $w(t)$ satisfies the inequality:

$$
\|w\|_{C\left([0, T] ; H^{2-\epsilon}(\Omega)\right)}^{2} \leq C(T)\left\|\frac{\partial}{\partial \nu} w_{t}\right\|_{L^{2}\left(\Sigma_{T}\right)}^{2} \quad \text { for } 0<\epsilon<\frac{1}{2} .
$$

Moreover, $C(T)>0$ is independent of the parameter $\gamma>0$.

Proof: By Proposition 2.2, $w(t)$ satisfies the desired inequality with the constant $C(T, \gamma)$ possibly dependent on $\gamma$. Assume (2.16) does not hold. Then there exists a sequence, $\left\{w_{\gamma}(t)\right\}_{\gamma \rightarrow 0^{+}}$, such that $w_{\gamma}(t)$ satisfies system (1.1) with $u=$ $-\frac{\partial}{\partial \nu} w_{\gamma, t}$ and

$$
\begin{aligned}
& \left\|\frac{\partial}{\partial \nu} w_{\gamma, t}\right\|_{L^{2}\left(\Sigma_{T}\right)}^{2} \rightarrow 0 \quad \text { as } \gamma \rightarrow 0^{+}, \\
& \left\|w_{\gamma}\right\|_{C\left([0, T] ; H^{2-\epsilon}(\Omega)\right)}^{2}=1 \quad \text { for each } \gamma .
\end{aligned}
$$

Then each $w_{\gamma}(t)$ satisfies (2.14). Therefore, as $\gamma \rightarrow 0^{+},\left\{w_{\gamma}(t), w_{\gamma, t}(t)\right\}$ remains in a bounded set of $C\left([0, T] ; H^{2}(\Omega) \times L^{2}(\Omega)\right)$. Hence, there exists a subsequence, also denoted by $\left\{w_{\gamma}(t)\right\}$, with

$$
\begin{aligned}
& w_{\gamma} \stackrel{w^{*}}{\longrightarrow} w \quad \text { in } L^{\infty}\left(0, T ; H^{2}(\Omega)\right) \\
& w_{\gamma, t} \stackrel{w^{*}}{\longrightarrow} w_{t} \quad \text { in } \quad L^{\infty}\left(0, T ; L^{2}(\Omega)\right)
\end{aligned}
$$

ESAIM: Proc., Vol. 4, 1998, 137-152 
Passing the limit as $\gamma \rightarrow 0^{+}$on system (1.1), we find that $w(t)$ must satisfy (see [10]):

$$
\begin{array}{rll}
w_{t t}+\Delta^{2} w=0 & \text { in } & Q_{T} \\
w=\Delta w=0 & \text { on } & \Sigma_{T} \\
\frac{\partial}{\partial \nu} w_{t}=0 & \text { on } & \Sigma_{T}
\end{array}
$$

Since $H^{2-\epsilon}(\Omega) \subset \subset H^{2}(\Omega)$, a result of Simon [14] gives

$$
w_{\gamma}(t) \rightarrow w(t) \quad \text { strongly in } C\left([0, T] ; H^{2-\epsilon}(\Omega)\right)
$$

Thus, by (2.17) and (2.20),

$$
\|w\|_{C\left([0, T] ; H^{2-\epsilon}(\Omega)\right)}^{2}=1 .
$$

Introducing the change of variable, $\psi \equiv w_{t}, \psi$ must satisfy the overdetermined system,

$$
\begin{array}{rll}
\psi_{t t}+\Delta^{2} \psi=0 & \text { in } & Q_{T} \\
\psi=\Delta \psi=0 & \text { on } & \Sigma_{T} \\
\frac{\partial}{\partial \nu} \psi=0 & \text { on } & \Sigma_{T}
\end{array}
$$

Therefore, [11] (Corollary 3.2, p. 256) states that $\psi \equiv 0$, contradicting (2.21). Hence, (2.16) must be valid with $C(T)$ independent of $\gamma$.

\section{Extensions to More Complex Systems}

Up to this point, the focus has been on a single Kirchhoff plate equation because it is easier to give the flavor of the challenges in obtaining robust stability results. Needless to say, as the systems under consideration become more complicated and include more complex dynamics rather than focusing on a single element, new problems must be addressed.

Extensions of these ideas from the Kirchhoff plate to a cylindrical shell is a perfect illustration of this phenomenon. A thin, isotropic, homogeneous cylindrical shell of constant thickness $h$, radius $a$, and length $L$ is considered. The displacement vector at time $t$ of a point $(\theta, x)$ on the neutral surface of the shell is denoted by $\{u(\theta, x, t), v(\theta, x, t), w(\theta, x, t)\}$ where $u(\theta, x, t)$ and $v(\theta, x, t)$ are the in-plane displacements along the axes of the neutral surface and $w(\theta, x, t)$ is the normal displacement of $(\theta, x)$. By including the effects of rotational inertia in a known model of the cylinder (see $[15,13])$, the linear system describing the 
vibrations of the cylinder can be found:

$$
\begin{array}{r}
u_{t t}=u_{x x}+\frac{1-\mu}{2 a^{2}} u_{\theta \theta}+\frac{1+\mu}{2 a^{2}} v_{\theta x}+\frac{\mu}{a} w_{x} \\
\left(1-\frac{\gamma}{a^{2}}\right) v_{t t}=\left(\frac{1-\mu}{2}\right)\left(1+\frac{D}{a^{2}}\right) v_{x x}+\frac{1}{a^{2}}\left(1+\frac{D}{a^{2}}\right) v_{\theta \theta} \\
-\frac{\gamma}{a^{2}} w_{\theta t t}-\frac{D}{a^{2}}\left(w_{\theta x x}+w_{\theta \theta \theta}\right) \\
+\left(\frac{1+\mu}{2}\right) u_{\theta x}+\frac{1}{a^{2}} w_{\theta} \\
w_{t t}-\gamma\left(w_{x x t t}+\frac{1}{a^{2}} w_{\theta \theta t t}\right)=D\left(w_{x x x x}+\frac{2}{a^{2}} w_{\theta \theta x x}+\frac{1}{a^{2}} w_{\theta \theta \theta \theta}\right) \\
-\frac{\gamma}{a^{2}} v_{\theta t t}-\frac{D}{a^{2}}\left(v_{\theta x x}+\frac{1}{a^{2}} v_{\theta \theta \theta}\right) \\
-\frac{1}{a}\left(\frac{1}{a}\left(v_{\theta}+w\right)+\mu u_{x}\right)
\end{array}
$$

subject to the initial conditions,

$$
\begin{gathered}
(u(\theta, x, 0), v(\theta, x, 0), w(\theta, x, 0))=\left(u_{0}(\theta, x), v_{0}(\theta, x), w_{0}(\theta, x)\right) \\
\left.\left(u_{t}(\theta, x, 0), v_{t}(\theta, x, 0), w_{t}(\theta, x, 0)\right)=\left(u_{1}(\theta, x), v_{1} \theta, x\right), w_{1}(\theta, x)\right)
\end{gathered}
$$

As for the Kirchhoff plate, the parameter $0<\gamma<1$ is proportional to the thickness and, therefore, assumed to be small. Additionally, $0<\mu<\frac{1}{2}$ is Poisson's ratio and $D$ measures the bending stiffness of the cylinder.

Boundary conditions imposed on the model will assume clamping at one end with velocity feedback acting as forces, moments and torques at the other:

$$
u(\theta, 0, t)=v(\theta, 0, t)=w(\theta, 0, t)=w_{x}(\theta, 0, t)=0
$$

and at $x=L$,

$$
\begin{aligned}
u_{x}+\frac{\mu}{a}\left(v_{\theta}+w\right) & =-u_{t} \\
\left(\frac{1-\mu}{2}\right)\left[v_{x}+\frac{1}{a} u_{\theta}+\frac{D}{a^{2}}\left(v_{x}-2 w_{\theta x}\right)\right] & =-2 v_{t}+w_{\theta t} \\
D\left[\left(\frac{1-\mu}{2 a^{2}}\right) v_{x}+\left(\frac{1+\mu}{2 a^{2}}\right) v_{\theta x}-w_{x x x}-\frac{1}{a^{2}} w_{x \theta \theta}+\gamma w_{x t t}-\left(\frac{1-\mu}{a^{2}}\right) w_{\theta x}\right] & =-v_{\theta t}+w_{\theta \theta t}-w_{t} \\
D\left(-w_{x x}+\frac{\mu}{a^{2}}\left(v_{\theta}-w_{\theta \theta}\right)\right) & =w_{x t}
\end{aligned}
$$

With this formulation, the model for the cylinder consists essentially of two wave equations, i.e., a two-dimensional system of elasticity, for the in-plane displacements and a Kirchhoff plate equation, including terms accounting for rotational inertia, for the normal displacement.

Wellposedness for the cylindrical shell which includes rotational inertia as well as its corresponding limit problem have been established in [13] and [12], respectively, and are stated in the following theorems.

ESAIM: Proc., Vol. 4, 1998, 137-152 
Theorem 3.1 $[13]$ (Wellposedness on $\left[\left(H^{1}(\Omega)\right)^{2} \times H^{2}(\Omega)\right] \times\left[\left(L^{2}(\Omega)\right)^{2} \times H^{1}(\Omega)\right]$.) Assume $\left(u_{0}, v_{0}, w_{0}\right) \in\left(H^{1}(\Omega)\right)^{2} \times H^{2}(\Omega),\left(u_{1}, v_{1}, w_{1}\right) \in\left(L^{2}(\Omega)\right)^{2} \times H^{1}(\Omega)$ satisfy:

$$
u_{0}=v_{0}=w_{0}=\frac{\partial}{\partial x} w_{0}=0 \quad \text { at } x=0 .
$$

Then there exists an unique solution (in the sense of distributions)

$$
\left\{(u, v, w),\left(u_{t}, v_{t}, w_{t}\right)\right\} \in C\left([0, T] ;\left[\left(H^{1}(\Omega)\right)^{2} \times H^{2}(\Omega)\right] \times\left[\left(L^{2}(\Omega)\right)^{2} \times H^{1}(\Omega)\right]\right)
$$

satisfying system (3.1-3.4).

Theorem 3.2 [12] (Wellposedness of the Limit Problem.)

Assume $\left(u_{0}, v_{0}, w_{0}\right) \in\left(H^{1}(\Omega)\right)^{2} \times H^{2}(\Omega),\left(u_{1}, v_{1}, w_{1}\right) \in\left(L^{2}(\Omega)\right)^{3}$ satisfy (3.5). Then there exists an unique solution (in the sense of distributions)

$$
\left\{(u, v, w),\left(u_{t}, v_{t}, w_{t}\right)\right\} \in C\left([0, T] ;\left[\left(H^{1}(\Omega)\right)^{2} \times H^{2}(\Omega)\right] \times\left(L^{2}(\Omega)\right)^{3}\right)
$$

satisfying the limiting system of (3.1-3.4) when $\gamma \rightarrow 0^{+}$.

With the above wellposedness results in mind, the energy of the system, defined by

$$
\begin{aligned}
E_{\gamma}(t) \equiv \frac{1}{2} \int_{\Omega} & {\left[u_{x}^{2}+\frac{2 \mu}{a} u_{x}\left(v_{\theta}-w_{\theta \theta}\right)+\frac{1}{a^{2}}\left(v_{\theta}-w_{\theta \theta}\right)^{2}+\left(\frac{1-\mu}{2}\right)\left(v_{x}+\frac{1}{a} u_{\theta}\right)^{2}\right] d \Omega } \\
& +\frac{D}{2} \int_{\Omega}\left[w_{x x}^{2}+\frac{2 \mu}{a^{2}} w_{x x}\left(v_{\theta}-w_{\theta \theta}\right)+\frac{1}{a^{4}}\left(v_{\theta}-w_{\theta \theta}\right)^{2}+\left(\frac{1-\mu}{2 a^{2}}\right)\left(v_{x}-2 w_{\theta x}\right)^{2}\right] d \Omega \\
& +\frac{1}{2} \int_{\Omega}\left(u_{t}^{2}+v_{t}^{2}+w_{t}^{2}\right) d \Omega+\frac{\gamma}{2} \int_{\Omega}\left[w_{x t}^{2}+\frac{1}{a^{2}}\left(v_{t}-w_{\theta t}\right)^{2}\right] d \Omega
\end{aligned}
$$

can be shown to be equivalent to $\left[\left(H^{1}(\Omega)\right)^{2} \times H^{2}(\Omega)\right] \times\left[\left(L^{2}(\Omega)\right)^{2} \times H^{1}(\Omega)\right]$ for fixed $\gamma>0$. If $\gamma=0, E_{0}(t)$ is equivalent to $\left.\left[\left(H^{1}(\Omega)\right)^{2} \times H^{2}(\Omega)\right] \times\left(L^{2}(\Omega)\right)^{3}\right)$.

The question of stabilization for the cylindrical shell described by (3.1-3.4) has been addressed in [13], while its corresponding limiting system when $\gamma=0$ is discussed in [12]. Clearly, establishing that stability estimates for this system are independent of $\gamma>0$ is more problematic than for the case of the Kirchhoff plate. The high degree of coupling within this system alone is of significant concern.

With control active, the energy of the system can be shown to be dissipative, but more work must be done to show the energy decays exponentially. As for the plate equation, to establish uniform stability for the cylinder, it suffices to prove:

Lemma 3.1 (Stability Estimate.) Let $\mathbf{u}=(u, v, w)$ be the solution to (3.1-3.4). Then for some $T$ sufficiently large, $\mathbf{u}(t)$ satisfies the inequality:

$$
E_{\gamma}(T) \leq C(T) \int_{0}^{T} \int_{\partial \Omega_{L}}\left(\left|u_{t}\right|^{2}+\left|v_{t}\right|^{2}+\left|w_{t}\right|^{2}+\left|w_{x t}\right|^{2}+\left|w_{\theta t}\right|^{2}\right) d \Omega_{L} d t,
$$

where $C(T)$ is independent of $\gamma$ and $\partial \Omega_{L}=\{(\theta, x, t) \mid x=L\}$. 
A stability estimate of the form in (3.9) is established in [13], however, the constant $C(T)$ is not shown to be independent of $\gamma$. To prove the inequality in Lemma 3.1 is valid, the system is rewritten in an equivalent variational formulation. Within this variational framework, the same multipliers that appear in work for the wave and plate equations are used to establish preliminary estimates. These multipliers include: $\nabla \mathbf{u h}$ (equivalently, $\mathbf{h} \cdot \nabla u, \mathbf{h} \cdot \nabla v, \mathbf{h} \cdot \nabla w$ ) and $\mathbf{u}$ (equivalently, $u, v, w$ ) and are used to show:

Lemma 3.2 (Preliminary Estimate.) Let $\mathbf{u}=(u, v, w)$ be the solution to (3.13.4). Then for some $T$ sufficiently large, $\mathbf{u}(t)$ satisfies the inequality:

$$
\begin{aligned}
E_{\gamma}(T) \leq & C(T)(1+\gamma)\left\{\int_{0}^{T} \int_{\partial \Omega_{L}}\left(\left|u_{t}\right|^{2}+\left|v_{t}\right|^{2}+\left|w_{t}\right|^{2}+\left|w_{x t}\right|^{2}+\left|w_{\theta t}\right|^{2}\right) d \Omega_{L} d t\right. \\
& +\int_{0}^{T} \int_{\partial \Omega_{L}}\left(\left|w_{\theta \theta}\right|^{2}+\left|w_{\theta x}\right|^{2}+\left|v_{\theta}\right|^{2}+\left|u_{\theta}\right|^{2}+|u|^{2}+|v|^{2}+|w|^{2}\right) d \Omega_{L} d t \\
& \left.+\int_{0}^{T} \int_{\Omega} w^{2} d \Omega d t\right\}
\end{aligned}
$$

where $C(T)$ is independent of $\gamma$.

Removal of the second two double integrals is the critical step in establishing (3.9). As for the Kirchhoff plate, traces of the solution on the boundary and lower order terms create the primary difficulties and must be bounded in terms of the norms of the controls.

To remove the traces which are not lower order with respect to the energy of the system, sharp trace regularity estimates must be used. Because this system is a coupled system combining a two-dimensional system of elasticity and a Kirchhoff plate equation, appropriate trace estimates are required. As discussed in previous example, the Kirchhoff plate is a known quantity. However, note that the boundary conditions for $w$ are not the same as those in problem (1.1). Thus, while Proposition 2.1 cannot be applied, however, sharp trace regularity estimates for the Kirchhoff plate with free boundary conditions can be found in [9]. In addition, analogous trace results now exist for the system of elasticity as discussed in [1]. Combining these estimates results in the following proposition.

Proposition 3.1 Let $\mathbf{u}=(u, v, w)$ be the solution to (3.1-3.4) and $0<\alpha<$ $T / 2$. Then $\mathbf{u}(t)$ satisfies the inequality:

$$
\begin{gathered}
\int_{\alpha}^{T-\alpha} \int_{\partial \Omega_{L}}\left(\left|w_{\theta \theta}\right|^{2}+\left|w_{\theta x}\right|^{2}+\left|v_{\theta}\right|^{2}+\left|u_{\theta}\right|^{2}+|u|^{2}+|v|^{2}+|w|^{2}\right) d \Omega_{L} d t \\
\leq C(T)(1+\gamma)\left\{\int_{0}^{T} \int_{\partial \Omega_{L}}\left(\left|u_{t}\right|^{2}+\left|v_{t}\right|^{2}+\left|w_{t}\right|^{2}+\left|w_{x t}\right|^{2}+\left|w_{\theta t}\right|^{2}\right) d \Omega_{L} d t\right. \\
\left.+\int_{0}^{T} \int_{\Omega} w^{2} d \Omega d t\right\}
\end{gathered}
$$

where $C(T)$ is independent of $\gamma$.

ESAIM: Proc., Vol. 4, 1998, 137-152 
Thus, with this proposition, the final issue is to remove the lower order terms. This is done, as before, through the use of a compactness/uniqueness argument. To reach the conclusion of (3.9), the following lemma must be proven.

Lemma 3.3 Let $\mathbf{u}=(u, v, w)$ be the solution to (3.1-3.4). Then $\mathbf{u}(t)$ satisfies the estimate:

$$
\begin{aligned}
& \|u\|_{C\left([0, T] ; H^{1-\epsilon}(\Omega)\right)}^{2}+\|v\|_{C\left([0, T] ; H^{1-\epsilon}(\Omega)\right)}^{2}+\|w\|_{C\left([0, T] ; H^{2-\epsilon}(\Omega)\right)}^{2} \\
& \quad \leq C(T) \int_{0}^{T} \int_{\partial \Omega_{L}}\left(\left|u_{t}\right|^{2}+\left|v_{t}\right|^{2}+\left|w_{t}\right|^{2}+\left|w_{\theta t}\right|^{2}+\left|w_{x t}\right|^{2}\right) d \Omega_{L} d t
\end{aligned}
$$

where $C(T)>0$ is independent of $\gamma$.

Proof: The estimate in (3.12) is established in [13] with $C(T)$ possibly dependent upon $\gamma$. To prove that $C(T)$ is independent of $\gamma$, the proof proceeds by contradiction. Assume that $C(T)$ is not independent of $\gamma$. Then there exists a sequence, $\left\{\gamma_{n}\right\}_{n=1}^{\infty}, \gamma_{n} \rightarrow 0^{+}$, such that $\left\{\mathbf{u}_{\gamma}, \mathbf{u}_{\gamma, t}\right\}$ satisfies (3.1-3.4) with

$$
\begin{aligned}
\left\|u_{\gamma_{n}}\right\|_{C\left([0, T] ; H^{1-\epsilon}(\Omega)\right)}^{2}+\left\|v_{\gamma_{n}}\right\|_{C\left([0, T] ; H^{1-\epsilon}(\Omega)\right)}^{2}+\left\|w_{\gamma_{n}}\right\|_{C\left([0, T] ; H^{2-\epsilon}(\Omega)\right)}^{2} & =1 \\
\int_{0}^{T} \int_{\partial \Omega_{L}}\left(\left|u_{\gamma_{n}, t}\right|^{2}+\left|v_{\gamma_{n}, t}\right|^{2}+\left|w_{\gamma_{n}, t}\right|^{2}+\left|w_{\gamma_{n}, \theta t}\right|^{2}+\left|w_{\gamma_{n}, x t}\right|^{2}\right) d \Omega_{L} d t & \rightarrow 0 \\
\text { as } \gamma_{n} & \rightarrow 0^{+}
\end{aligned}
$$

Then each $\mathbf{u}_{\gamma}(t)$ satisfies the inequality:

$$
\begin{aligned}
E_{\gamma}(T) & \leq C(T)(1+\gamma)\left\{\int _ { 0 } ^ { T } \int _ { \partial \Omega _ { L } } \left(\left|u_{t}\right|^{2}+\left|v_{t}\right|^{2}+\left|w_{t}\right|^{2}+\left|w_{x t}\right|^{2}+\right.\right. \\
& \left.+\left|w_{\theta t}\right|^{2}\right) d \Omega_{L} d t+\left\|u_{\gamma_{n}}\right\|_{C\left([0, T] ; H^{1-\epsilon}(\Omega)\right)}^{2}+\left\|v_{\gamma_{n}}\right\|_{C\left([0, T] ; H^{1-\epsilon}(\Omega)\right)}^{2}+ \\
& \left.+\left\|w_{\gamma_{n}}\right\|_{C\left([0, T] ; H^{2-\epsilon}(\Omega)\right)}^{2}\right\} .
\end{aligned}
$$

Therefore, as $\gamma_{n} \rightarrow 0^{+},\left\{\mathbf{u}_{\gamma_{n}}, \mathbf{u}_{\gamma, t}\right\}$ remains in a bounded set of $\left[\left(H^{1}(\Omega)\right)^{2} \times\right.$ $\left.H^{2}(\Omega)\right] \times\left(L^{2}(\Omega)\right)^{3}$. Hence, there exists a subsequence, also denoted by $\left\{\mathbf{u}_{\gamma_{n}}, \mathbf{u}_{\gamma, t}\right\}$ with

$$
\begin{array}{ll}
\mathbf{u}_{\gamma_{n}} \stackrel{w}{\longrightarrow} \mathbf{u} & \text { in } \\
\mathbf{u}_{\gamma_{n}, t} \stackrel{w}{\longrightarrow} \mathbf{u}_{t} & \quad \text { in } C\left([0, T] ;\left(H^{1}(\Omega)\right)^{2} \times H^{2}(\Omega)\right),
\end{array}
$$

Thus, by [14] and Sobolev imbeddings,

$$
\mathbf{u}_{\gamma_{n}} \stackrel{s}{\longrightarrow} \mathbf{u} \quad \text { in } C\left([0, T] ;\left(L^{2}(\Omega)\right)^{2} \times H^{1}(\Omega)\right) .
$$

This is sufficient regularity to justify passing with the limit as $\gamma_{n} \rightarrow 0^{+}$and therefore $\mathbf{u}(t)$ must satisfy

$$
\|u\|_{C\left([0, T] ; H^{1-\epsilon}(\Omega)\right)}^{2}+\|v\|_{C\left([0, T] ; H^{1-\epsilon}(\Omega)\right)}^{2}+\|w\|_{C\left([0, T] ; H^{2-\epsilon}(\Omega)\right)}^{2}=1
$$

and the overdetermined problem composed of system (3.1) with $\gamma=0$ and the boundary conditions,

$$
u(\theta, 0, t)=v(\theta, 0, t)=w(\theta, 0, t)=w_{x}(\theta, 0, t)=0
$$


and at $x=L$,

$$
\begin{aligned}
u_{x}+\frac{\mu}{a}\left(v_{\theta}+w\right) & =0 \\
\left(\frac{1-\mu}{2}\right)\left[v_{x}+\frac{1}{a} u_{\theta}+\frac{D}{a^{2}}\left(v_{x}-2 w_{\theta x}\right)\right] & =0 \\
D\left[\left(\frac{1-\mu}{2 a^{2}}\right) v_{x}+\left(\frac{1+\mu}{2 a^{2}}\right) v_{\theta x}-w_{x x x}-\frac{1}{a^{2}} w_{x \theta \theta}-\left(\frac{1-\mu}{a^{2}}\right) w_{\theta x}\right] & =0 \\
D\left(-w_{x x}+\frac{\mu}{a^{2}}\left(v_{\theta}-w_{\theta \theta}\right)\right) & =0 \\
u_{t}=v_{t}=w_{t}=w_{\theta t}=w_{x t} & =0
\end{aligned}
$$

Unique continuation results for cylindrical shells have only recently become available. Results in $[12,13]$ are proven based on the strength of Carleman estimates. In particular, with regard to the above overdetermined problem, [12] established that if $\mathbf{u}(t)$ satisfies system (3.1) with $\gamma=0$ together with the boundary conditions in (3.19), then $\mathbf{u}(t) \equiv 0$, contradicting (3.17). Thus, $C(T)$ must be independent of $\gamma$.

With the result of Lemma 3.3, the desired stability estimate (3.9), with constant independent of the parameter $\gamma$, is obtained.

\section{Conclusions}

Robustness of feedback with respect to thickness can be extended to include highly coupled systems. However, the arguments depend critically on the problem under consideration and, thus, these techniques must be applied on a case by case basis. Particularly of importance is the reliance of the proofs on unique continuation results-results which largely do not exist for complex systems. But these arguments do permit two physical models, which may have very different mathematical properties, to be addressed simultaneously, when the second system is the limit case for the first.

\section{References}

[1] M. A. Horn. "Implications of sharp trace regularity results on boundary stabilization of the system of linear elasticity," Journal of Mathematical Analysis and Applications, submitted.

[2] M. A. Horn. "Uniform decay rates for the solutions to the Euler-Bernoulli plate equation with boundary feedback acting via bending moments," Differential and Integral Equations, 5 (5): 1121-1150, 1992.

[3] M. A. Horn. "Uniform stabilization of the Kirchhoff plate equation with boundary conditions containing moments of inertia," Journal of Mathematical Systems, Estimation and Control, 4 (1): 39-65, 1994. 
[4] M. A. Horn and I. Lasiecka. "Asymptotic behavior with respect to thickness of boundary stabilizing feedback for the Kirchhoff plate," Journal of Differential Equations, 114 (1.2):396-433, 1994.

[5] I. Lasiecka. "Exponential decay rates for the solutions of Euler-Bernoulli equations with boundary dissipation occurring in the moments only," Journal of Differential Equations, 95 (1):169-182, 1992.

[6] I. Lasiecka and R. Triggiani. "Exact controllability and uniform stabilization of Euler-Bernoulli equations with boundary control only in $\left.\Delta w\right|_{\Sigma}, "$ Boll. Un. Mat. Ital. B, 5 (7):665-702, 1991.

[7] I. Lasiecka and R. Triggiani. "Exact controllability and uniform stabilization of Kirchhoff plates with boundary control only on $\left.\Delta w\right|_{\Sigma}$ and homogeneous boundary displacement," Journal of Differential Equations, 93 (1):62-101, 1991.

[8] I. Lasiecka and R. Triggiani. "Regularity theory for a class of nonhomogeneous Euler-Bernoulli equations: A cosine operator approach," Boll. Un. Mat. Ital. B, 3 : 199-228, 1989.

[9] I. Lasiecka and R. Triggiani. "Sharp trace estimates of solutions to Kirchhoff and Euler-Bernoulli equations." Differential equations in Banach spaces (Bologna, 1991), Lecture Notes in Pure and Appl. Math., 148 :141-180, Marcel Dekker, New York, 1993.

[10] J. E. Lagnese. Boundary stabilization of thin plates, Society for Industrial and Applied Mathematics, Philadelphia, 1989.

[11] J.-L. Lions. Contrôlabilité exacte, perturbations et stabilization de systèmes distribués, Tome 1, Masson, Paris, 1989.

[12] C. A. McMillan. "Uniform stabilization of a thin cylindrical shell," preprint.

[13] C. A. McMillan. "Uniform stabilization of a thin cylindrical shell: The effects of rotational inertia," preprint.

[14] J. Simon. "Compact sets in the space $L_{p}(0, T ; B)$," Ann. Mat. Pura Appl., 146 (4): 65-96, 1987.

[15] W. Soedel. Vibrations of shells and plates, Marcel Dekker, New York, 1993. 